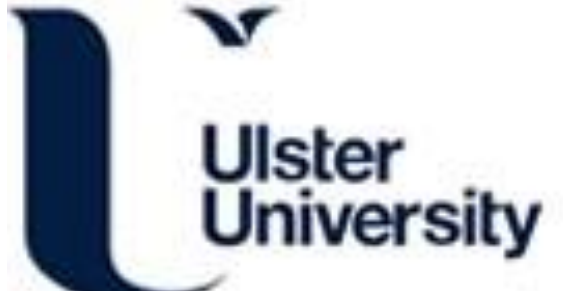

\section{Flexible and high-performance piezoresistive strain sensors based on carbon nanoparticles@polyurethane sponges}

Zhang, X., Xiang, D., Zhu, W., Zheng, Y., Harkin-Jones, E., Wang, P., Zhao, C., Li, H., Wang, B., \& Li, Y. (2020). Flexible and high-performance piezoresistive strain sensors based on carbon nanoparticles@polyurethane sponges. Composites Science and Technology, 200, [108437].

https://doi.org/10.1016/j.compscitech.2020.108437

Link to publication record in Ulster University Research Portal

\section{Published in:}

Composites Science and Technology

Publication Status:

Published (in print/issue): 10/11/2020

DOI:

10.1016/j.compscitech.2020.108437

\section{Document Version}

Author Accepted version

\section{General rights}

Copyright for the publications made accessible via Ulster University's Research Portal is retained by the author(s) and / or other copyright owners and it is a condition of accessing these publications that users recognise and abide by the legal requirements associated with these rights.

\section{Take down policy}

The Research Portal is Ulster University's institutional repository that provides access to Ulster's research outputs. Every effort has been made to ensure that content in the Research Portal does not infringe any person's rights, or applicable UK laws. If you discover content in the Research Portal that you believe breaches copyright or violates any law, please contact pure-support@ulster.ac.uk. 


\title{
Flexible and high-performance piezoresistive strain sensors based on carbon Nnanoparticles@Ppolyurethane sponges
}

Xuezhong Zhang ${ }^{\mathrm{a}, 1}$, Dong Xiang ${ }^{\mathrm{a}, 1, *}$ dxiang01@hotmail.com, Wanqiu Zhu ${ }^{\mathrm{a}}$, Yongfeng Zheng ${ }^{\mathrm{a}}$, Eileen Harkin-Jones $^{\mathrm{b}}$, Ping Wang ${ }^{\mathrm{a}}$, Chunxia Zhao ${ }^{\mathrm{a}}$, Hui Li ${ }^{\mathrm{a}}$, Bin Wang ${ }^{\mathrm{a}}$, Yuntao Li ${ }^{\mathrm{a}, * *}$ yuntaoli@swpu.edu.cn a School of New Energy and Materials, Southwest Petroleum University, Chengdu, 610500, China ${ }^{\mathbf{b}}$ School of Engineering, University of Ulster, Jordanstown, BT37 0QB, UK

* Corresponding author.

** Corresponding author.

${ }^{1}$ These authors contributed equally to this work.

\begin{abstract}
In this work, flexible and high-performance piezoresistive strain sensors were fabricated by simple layer-by-layer electrostatic self-assembly of carbon nanoparticles on commercial polyurethane (PU) sponges. It was shown that the sponge-based strain sensors exhibited obviously positive and negative piezoresistive characteristics under tensile and compressive strains, respectively. The alternate assembly of carbon nanotubes (CNTs) and graphene nanoplatelets (GNPs) contributed to the construction of a more complete conductive network and significantly improved the sensing performance of the sensor due to the synergistic effect between CNTs and GNPs. Compared with the CNT@PU and CNT/GNP@PU sponge strain sensors, the CNT/GNP/CNT@PU sensor had a larger strain detection range and higher linearity. Besides, the CNT/GNP/CNT@PU sponge strain sensor showed high sensitivity $(\mathrm{GF}=43,000$ at $60 \%$ tensile strain and $\mathrm{GF}=-1.1$ at $50 \%$ compressive strain), responsive capability to very small strain (0.05\%) and outstanding stability during 3000 loading cycles. Due to its excellent sensing performance, the CNT/GNP/CNT@PU sensor enabled monitoring of various physiological activities, including finger movements, wrist bending and walking etc. In addition, a $5 \times 5$ sensor array based on the sponge-based strain sensor was prepared to achieve accurate identification of weight distribution. This study provides valuable information for the development of flexible strain sensors with high-performance and low-cost.
\end{abstract}




\section{Introduction}

In the past decade, flexible strain sensors have been increasingly used in health monitoring [1], human-computer interaction [2], wearable electronic devices [3], smart home appliances and soft robots [4]. Compared with traditional metal or semiconductor strain sensors, flexible strain sensors have attracted more attention due to their high stretchability, large strain response range, and high sensitivity. These flexible strain sensors are mainly composed of flexible substrate and conductive nanofillers. The flexible substrates include polyurethane (PU) [5], polydimethylsiloxane (PDMS) [6], natural rubber (NR) [7], styrene-butadiene-styrene block copolymer (SBS) [8 ] etc. Conductive nanofillers usually include carbon nanomaterials [9-12] (carbon black, carbon nanotubes [13], graphene nanoplatelets [14]), metal nanomaterials (silver nanowires [1], silver nanoparticles [15], gold nanoparticles) and intrinsically conductive polymers (polyaniline [16], polypyrrole [17], polythiothene). Carbon nanomaterials are more widely used in the preparation of flexible strain sensors due to their low cost, excellent electrical conductivity and good processability. Carbon nanotubes (CNTs) have large aspect ratios and high electrical conductivity making them very suitable for use as the conductive nanofillers in flexible strain sensors [ 18]. However, CNTs are difficult to uniformly disperse in a polymer matrix due to surface inertness, which limits the sensitivity of the sensor [19]. By contrast, GNPs are apt to slip during deformation due to their special twodimensional structure, which improves the sensitivity of the sensor and reduces the strain response range [13].

Excellent strain sensors typically demand high sensitivity, large strain response range, good stability and the ability to accurately identify different strains. Recent studies have reported various flexible strain sensors with relatively high sensitivity and large strain ranges. Chen et al. [18] prepared a stretchable flexible strain sensor with high sensitivity (Gauge factor/GF $=1665.9$ at the strain of 100\%) using CNTs coated spandex fibers. Furthermore, the use of 1-pyridinecarboxylic acid (PCA) improved the dispersability of the carbon nanotubes and the interfacial adhesion between the polymer and nanofillers via non-covalent interactions, which enhanced the sensitivity of strain sensor $(\mathrm{GF}=14191.5)$ and resulted in a larger strain monitoring range $(>200 \%$ strain $)[19$ ]. Gong et al. [20] prepared a high-performance strain sensor by doping polystyrene (PS) nanoparticles into reduced graphene oxide (rGO) film. The nanoparticles functioned as insulators that separated the stacked rGO sheets and created partially connected conducting channels, thus significantly modified the resistance-changing behavior. Wang et al. [21] prepared a strain sensor with ultra-stretchability (920\% strain) and high sensitivity (GF $=129$ under the strain of 780-920\%) by embedding CNTs into electronic bandages using a swellingultrasonication treatment. Ryu et al. [22] prepared a highly stretchable ( $900 \%$ strain) and durable strain sensor based on a CNT decorated thermoplastic polyurethane fibrous network with aligned structure. Although these reported sensors have high sensitivity at large strains and good flexibility, generally they can only effectively identify tensile strain and have relatively low sensitivity at small strains. Therefore, the application of these strain sensors in real life is limited [23].

Foam or sponge-based strain sensors have a better ability to identify strains in different ways [24]. Very recently, Wang et al. [25] developed an elastic and versatile piezoresistive strain sensor by encapsulating carbonized phenolic foam (PFF) with polydimethylsiloxane (PDMS). The strain sensor could detect various strains (stretching, compression and three-point bending), but it was difficult to effectively monitor large strains due to limitations in flexibility. Wu et al. [26] prepared a carbon black/polyurethane sponge strain sensor with a microcrack mechanism using electrostatic assembly. Although the strain sensor exhibited good stability $(>5000$ cycles), it could only effectively monitor compressive strains. Qin et al. [27] reported a method to prepare a rGO aerogel-based sensor by introducing water-soluble polyimide (PI). The synergy between the PI and rGO endowed the elastomer with excellent stretchability, electrical conductivity, compression sensitivity and durability. Zeng et al. [24] prepared a nanocomposite strain sensor with a wide linear sensing region based on 
anisotropic rGO foam and PDMS by unidirectional freeze-drying. The most commonly reported preparation method to date for foam or sponge-based strain sensors is freeze-drying. However, due to the high cost and complex processes, freeze-drying technology is difficult for large-scale manufacturing of sensors.

In this work, we are aiming to develop a simple strategy to fabricate a high-performance flexible strain sensor based on carbon nanoparticles (CNTs and GNPs) and commercial PU sponges. The oppositely charged CNTs and GNPs were alternately deposited on the surface of the sponge backbones via a layer-by-layer electrostatic self-assembly method. The synergistic effect between CNTs and GNPs on the electrical and sensing performance of the strain sensors were systematically investigated. It was shown that the CNT/GNP/CNT@PU sensor prepared in this work exhibited a better comprehensive sensing performance with the advantages of simplicity, low cost and ease of production, compared to the recently reported sensors.

\section{Material and methods}

\subsection{Materials}

CNTs (NC7000) with an average diameter around $9.5 \mathrm{~nm}$ and a length of $1.5 \mu \mathrm{m}$ were obtained from Nanocyl S.A (Belgium) [28]. The purity and density of CNTs are $90 \%$ and $1.85 \mathrm{~g} / \mathrm{cm}^{3}$, respectively. GNPs with a lamellar size of $10 \mu \mathrm{m}$ and a mean thickness of $3 \mathrm{~nm}$ were supplied by Deyang carbonene Technology Co. Ltd (China). The purity and density of GNPs are $99.5 \%$ and $2.0 \mathrm{~g} / \mathrm{cm}^{3}$, respectively. Polyvinyl Pyrrolidone (PVP, KH-16), sodiumdodecylbenzenesulfonate (SDBS) were obtained from Chengdu Cologne Chemical Reagent Company. Polyurethane (PU) sponge (Bayer 302) was purchased from Jiangsu Yiruilong Co. Ltd. The N, N-di (2-aminoethyl)-perylene-3,4,9,10-tetracarboxylicdiimide (AE-PTDI) was supplied by Shanghai Aladdin BioChem Technology Co. Ltd.

\subsection{Preparation of sponge-based strain sensors}

As shown in Fig. 1a, CNTs, AE-PTDI, PVP were first dispersed in deionized water via ultra-sonication at $100 \mathrm{~W}$ and $40 \mathrm{kHz}$ for $1 \mathrm{~h}$ at room temperature to obtain a CNT suspension [29]. AE-PTDI performs noncovalent bond modification of CNTs by $\pi-\pi$ stacking with CNTs [30], which helps to positively charge CNTs without destroying the CNT structure and conductivity. Non-ionic PVP can further improve the dispersability of carbon nanoparticles by acting as a surfactant, and it facilitates the deposition of nanoparticles onto the sponge as a thickening agent. Fig. 1b shows the electronegativity changes of nanoparticles/PVP suspension tested by a Zeta potentiometer (Zeta PALS 190 Plus). The Zeta potential $(\zeta)$ of the CNT/PVP suspension after AE-PTDI modification increased from $-2.9 \mathrm{mV}$ to $+34.2 \mathrm{mV}$. The PU sponge was soaked in the CNT suspension at room temperature for $6 \mathrm{~h}$, and then dried to obtain the CNT@PU. GNPs, SDBS, PVP were also dispersed in deionized water via ultra-sonication at $100 \mathrm{~W}$ and $40 \mathrm{kHz}$ for $1 \mathrm{~h}$ at room temperature to obtain a GNP suspension. As an anionic surfactant, SDBS can be coated on the surface of GNPs to form a negatively charged layer [31]. The $\zeta$ of the GNP/PVP suspension after SDBS modification decreased from $-11.7 \mathrm{mV}$ to $-41.6 \mathrm{mV}$ (Fig. 1b). The negatively charged GNPs could be fixed on the positively charged CNT@PU by electrostatic self-assembly. Therefore, the CNT@PU was soaked in the GNP suspension at room temperature for another $6 \mathrm{~h}$, and then dried to obtain the CNT/GNP@PU. Then the CNT/GNP@PU was soaked in the CNT suspension at room temperature for $6 \mathrm{~h}$ again, and then dried to obtain the CNT/GNP/CNT@PU. The resulting PU sponges were connected to two silver wire electrodes with a distance of $15 \mathrm{~mm}$ using silver paste, and their top and bottom surfaces were encapsulated by PDMS (thickness $\approx 0.5 \mathrm{~mm}$ ) at $80{ }^{\circ} \mathrm{C}$ for $1 \mathrm{~h}$ to prepare the strain sensors, as shown in Fig. 1c. 
(a)

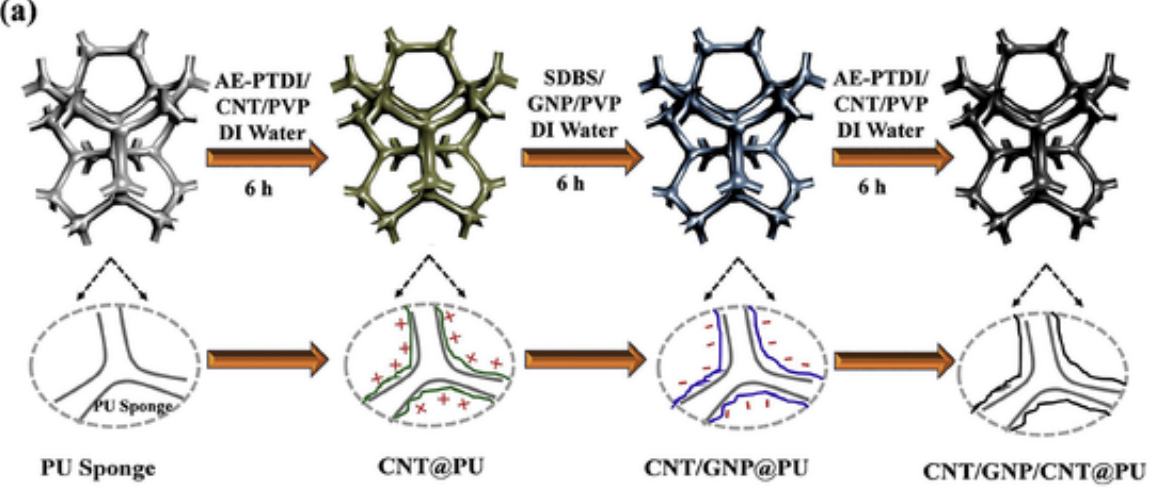

(b)

(c)
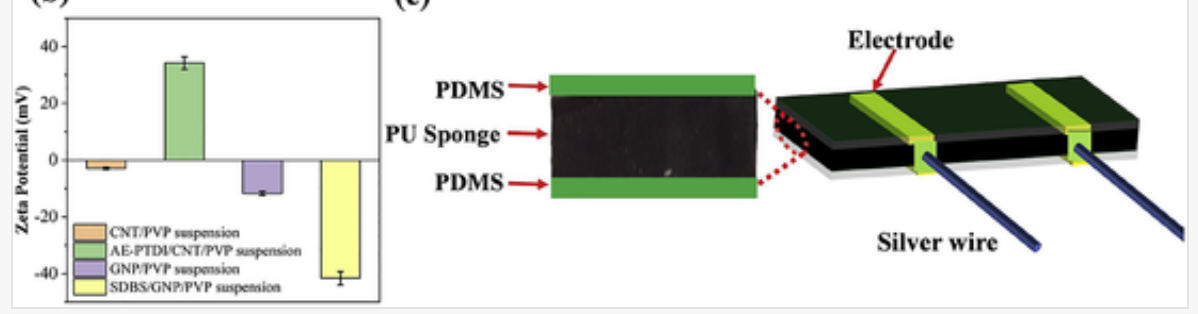

Schematic diagram for the preparation of the PU sponge-based strain sensors: (a) layer-by-layer electrostatic self-assembly, (b) Zeta potential of the nanoparticles/PVP suspension, (c) PDMS encapsulation.

\subsection{Characterization}

Morphology and structure: The surface morphology of the treated PU sponges was studied using a FEI Quanta $650 \mathrm{FEG}$ field emission scanning electron microscope (FESEM) at $30 \mathrm{kV}$ accelerating voltage. Apart from the pristine PU sponge, the samples were not gold sputtered before observation. Fourier-transform infrared spectroscopy (FTIR) was conducted using a WQF-520 FTIR spectrometer with a wavenumber range from 500 to $4000 \mathrm{~cm}^{-1}$. X-ray diffraction (XRD) analysis was conducted using a D8-Adcance instrument (Germany) and $\mathrm{Cu}-\mathrm{Ka}$ radiation with a scanning speed of $1 \% \mathrm{~min}$.

Electrical conductivity: The conductivity $(\sigma)$ of the sponge-based sensors was measured using a two-point method combined with a DC digital source meter (Tektronix PWS4323) and picoamp-meter (Keithley 6485) at $3 \mathrm{~V}$. The conductivity of the samples was calculated using Eq. (1):

$\sigma=\frac{I}{U} \times \frac{L}{S}$

where I and $\mathrm{U}$ are the current and voltage of the sample, $\mathrm{L}$ and $\mathrm{S}$ are the electrode distance and cross-sectional area of the sample, respectively.

Sensing performance: The sensing performance of the sponge-based strain sensors was studied by combining a universal tester (MTS CMT4104) and the above mentioned DC digital source meter and picoamp-meter at $3 \mathrm{~V}$. In order to more accurately characterize the relationship between strain and relative resistance change $\left(\Delta \mathrm{R} / \mathrm{R}_{0}\right)$, the same size samples $(50 \mathrm{~mm} \times 10 \mathrm{~mm} \times 5 \mathrm{~mm})$ were subjected to tensile and compression tests. The sensitivity of the sensor is usually expressed by a gauge factor (GF) calculated using Eq. (2): 


$$
\mathrm{GF}=\frac{\Delta R}{R_{0} \times \varepsilon}
$$

where $\varepsilon, \Delta \mathrm{R}$ and $\mathrm{R}_{0}$ represent the applied strain, resistance change under strain and initial resistance, respectively [30].

\section{Results and discussion}

\subsection{Morphology and structure}

A photograph of the CNT/GNP/CNT@PU sponge is shown in Fig. 2a. The pristine PU sponge has a smoother surface with a pore size of about $1 \mathrm{~mm}$ (Fig. 2b), compared with the treated PU sponge shown in Fig. 2c. The pristine PU sponge was alternately dipped into oppositely charged CNT/PVP and GNP/PVP suspensions, resulting in the electrostatic deposition of nanoparticles on the surface of sponge backbones (Fig. 2c). After the first electrostatic self-assembly, many positively charged CNTs were immobilized on the surface of the PU to form conductive paths (Fig. 2d). After the second electrostatic deposition process, a large number of negatively charged GNPs were successfully assembled on the CNT layer of the CNT@PU (from Fig. 2e) to enable longdistance electron transmission [32]. From Fig. 2f, the CNT/GNP/CNT@PU was obtained by another electrostatic self-assembly of CNTs on the surface of the CNT/GNP@PU. The 1-dimensional CNTs bridged the gaps between the sheet-like GNPs, further enhancing the conductive network on the sponge surface. More SEMSEM-images of the PUPU-sponge-based strain sensors are shown in Fig. S1 in Supporting Information.

alt-text: Fig. 2

Fig. 2 

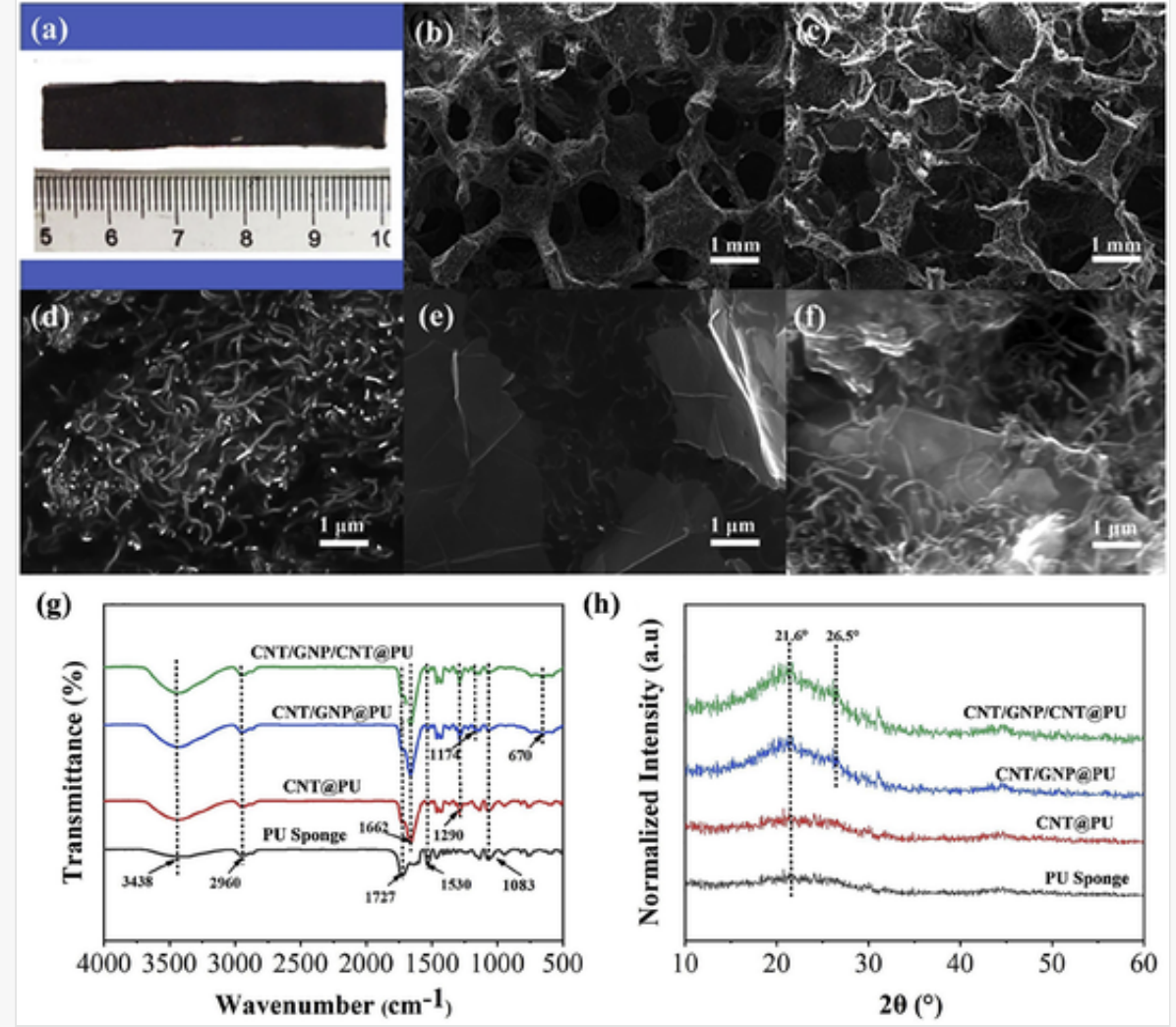

(a) Photograph of the CNT/GNP/CNT@PU sponge; SEM images of the pristine and treated PU sponges: (b) pristine PU sponge, (c, f) CNT/GNP/CNT@PU, (d) CNT@PU, (e) CNT/GNP@PU; (g) FTIR and (h) XRD spectra of the PU sponge, CNT@PU,CNT/GNP@PU and CNT/GNP/CNT@PU.

FTIR was performed to further verify that the nanoparticles were deposited on the PU sponge by electrostatic self-assembly [26], as shown in Fig. $2 \mathrm{~g}$. The peaks at $3438 \mathrm{~cm}^{-1}$ and $2960 \mathrm{~cm}^{-1}$ are attributed to the N-H and -C-H stretching vibration bands of PU [21]. The absorption peaks of the -H-N-COO- group are located at $1727 \mathrm{~cm}^{-1}$ and $1530 \mathrm{~cm}^{-1}$. The peak at $1083 \mathrm{~cm}^{-1}$ represents the stretching vibration of the $\mathrm{C}-O-\mathrm{C}$ group [33 ]. For the CNT@PU, the absorption peak at $1662 \mathrm{~cm}^{-1}$ is assigned to the $\mathrm{C}==\mathrm{O}$ stretching vibration of PVP, and the absorption peak at $1290 \mathrm{~cm}^{-1}$ is assigned to the $\mathrm{C}-\mathrm{N}$ stretching vibration of AE-PTDI, indicating that the modified CNTs were deposited on the PU sponge. For the CNT/GNP@PU, the absorption peaks at $1174 \mathrm{~cm}^{-1}$ and $670 \mathrm{~cm}^{-1}$ represent the stretching vibration peaks of $\mathrm{S}==\mathrm{O}$ and $\mathrm{S}-\mathrm{O}$ in SDBS, respectively, 
revealing the immobilization of modified GNPs on the sponges. In addition, the peaks at $1174 \mathrm{~cm}^{-1}$ and $670 \mathrm{~cm}^{-1}$ can be still observed for the CNT/GNP/CNT@PU, indicating that the subsequent electrostatic selfassembly process of CNTs did not destroy the previously deposited GNPs.

The XRD results of the sponge-based strain sensors also showed that the nanoparticles were deposited on the surface of the PU sponge, as shown in Fig. 2h. The PU sponge exhibited a broad diffraction peak around $21.6^{\circ}$, which was resulted from the irregular crystalline region of the hard segment of PU [32,34]. Generally, CNTs have a broad diffraction peak around $26^{\circ}$, but the addition of PVP makes it more difficult to observe the diffraction peaks of CNTs for the CNT@PU [26,27]. It is seen that the peak intensity of the diffraction peak for the CNT@PU is slightly stronger than that of the PU sponge. The rearrangement and crystallization of the molecular segment of the PVP on the PU sponge surface may result in this phenomenon [21]. Due to the special graphite sheet structure of GNPs, the GNPs have a clear diffraction peak at 26.5 [15]. The CNT/GNP@PU exhibited a characteristic diffraction peak of GNPs, demonstrating that GNPs were successfully deposited on the surface of the PU sponge. The XRD diffraction peak of the CNT/GNP/CNT@PU at 26.5 still existed, indicating that the subsequent electrostatic self-assembly process of CNTs did not remove the GNPs as stated earlier.

\subsection{Electrical properties}

The number of electrostatic self-assembly layers has a significant effect on the electrical conductivity of the sponge. As shown in Fig. 3a, the conductivity of the CNT/GNP/CNT@PU is 76\% and 663\% higher than that of the CNT/GNP@PU and CNT@PU, respectively. As illustrated in Fig. 3e, the increased number of layers facilitates the building of a more complete conductive network, which greatly reduces the resistance of the sponge. It can be clearly observed in Fig. $3 \mathrm{~b}$ that the current of sensors increases linearly with increasing voltage, which agrees well with Ohm's law. From Fig. S2, the conductivity of sponge-based sensors was not significantly changed within the temperature range of $20-60{ }^{\circ} \mathrm{C}$, indicating that it was independent of temperature. From Fig. $3 \mathrm{c}$, the current of sensors decreases with increasing tensile strain, showing a positive piezoresistive effect. However, the current increased when the sponge sensor was compressed, showing a negative piezoresistive effect (Fig. 3d). During the deformation process of the sponge-based strain sensor, the "destruction" and "reconstruction" of the conductive paths proceed simultaneously (Fig. 3f). In the tensile process, the destruction of the conductive network was dominated, increasing the tunneling distance between adjacent conductive nanoparticles $[33,34]$. When the sensors were compressed, more conductive paths were reconstructed, resulting in the increase of conductivity $[35,36]$.

alt-text: Fig. 3

Fig. 3 
(a)

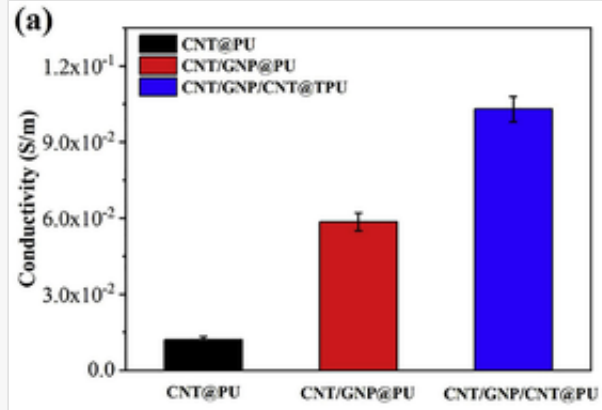

(b)
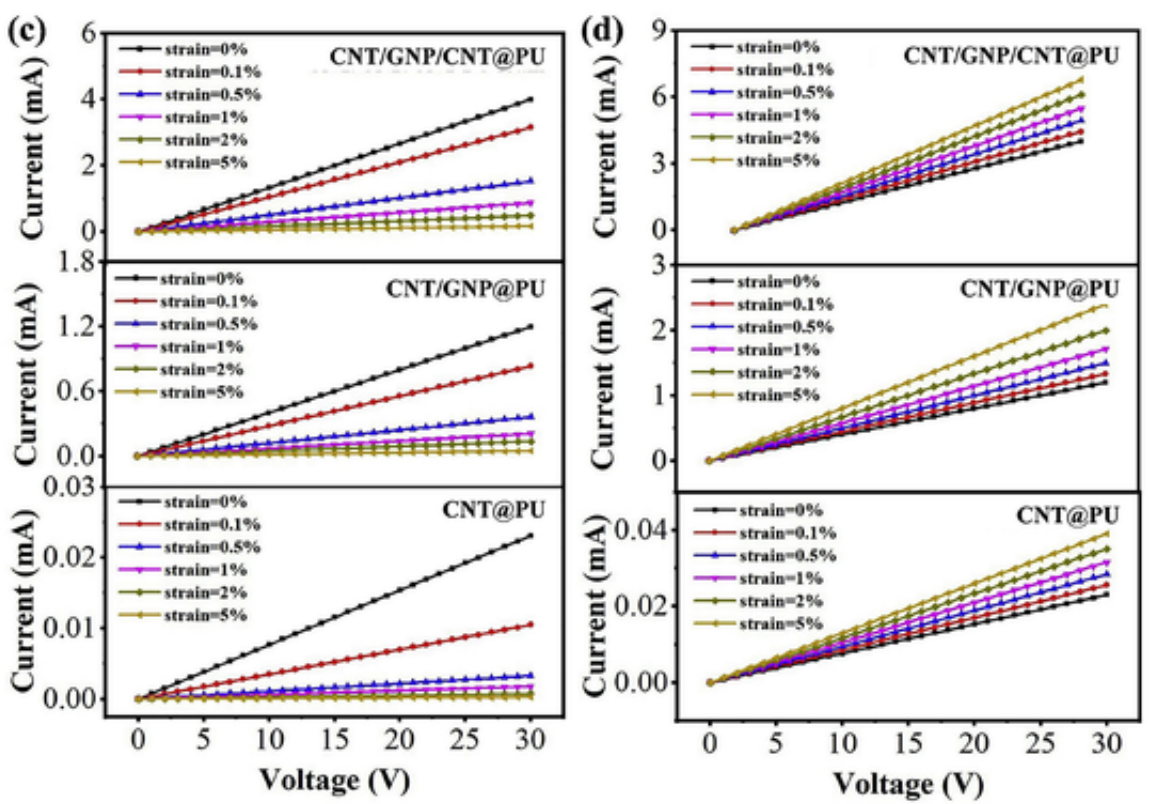

(e)

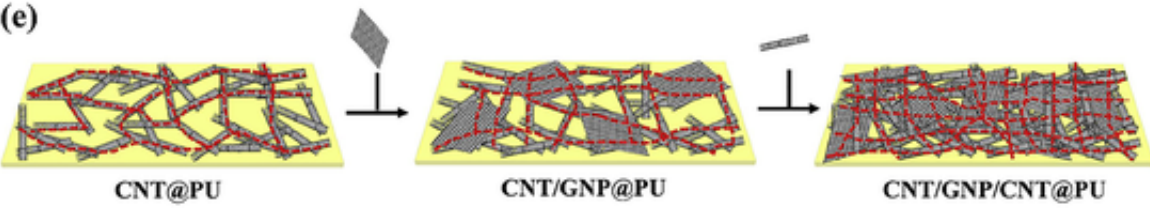

(f)
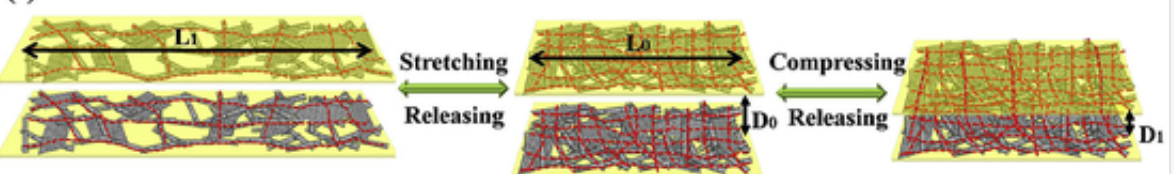

PU Sponge $=\mathrm{CNT}$ GNP

(a) Conductivity of the strain sensors versus the number of electrostatic self-assembly layers, (b) current-voltage curves of the strain sensors at different voltages; current-voltage curves of the strain sensors under different strains: (c) tensile strains $(0$, $0.1 \%, 0.5 \%, 1 \%, 2 \%, 5 \%)$, (d) compressive strains $(0,0.1 \%, 0.5 \%, 1 \%, 2 \%, 5 \%)$; schematic diagram for the evolution of conductive paths in the strain sensors: (e) with different number of self-assembly layers, (f) under different strains. 
As shown in Fig.4a and b, the CNT@PU sensor exhibited a relatively high sensitivity (GF = 7006 at $25 \%$ strain and $\mathrm{GF}=54,000$ at $40 \%$ strain) according to the $\Delta \mathrm{R} / \mathrm{R}_{0}$-strain curve, but its strain range was limited $(0 \sim 40 \%)$. As the number of electrostatic self-assembly layers increased, the sensitivity ( $G F=2393$ at $25 \%$ strain and $\mathrm{GF}=43,000$ at 60\% strain) of CNT/GNP/CNT@PU sensor reduced compared with that of the CNT@PU, but the workable strain range increased from $40 \%$ to $60 \%$. This can be attributed to the improvement of the conductive network on the sponge surface, which greatly enhances the ability of the conductive network to resist deformation. Although all the sponge-based strain sensors have high linearity $\left(\mathrm{R}^{2}>0.9\right)$ under low tensile strain $(0 \sim 25 \%)$, the linearity $\left(\mathrm{R}^{2}=0.98\right)$ of CNT/GNP/CNT@PU sensors with more robust conductive network is higher than that of CNT@PU $\left(\mathrm{R}^{2}=0.93\right)$ and CNT/GNP@PU $\left(\mathrm{R}^{2}=0.95\right)$. The linearity of the sponge-based strain sensors decreases with increasing strain $(\varepsilon>25 \%)$, while the linearity of the CNT/GNP/CNT@PU sensor $\left(\mathrm{R}^{2}=0.83\right)$ is still higher than that of CNT@PU $\left(\mathrm{R}^{2}=0.78\right)$ and CNT/GNP@PU $\left(\mathrm{R}^{2}=0.81\right)$. Due to the multi-layer conductive network structure constructed by the CNTs and GNPs, the CNT/GNP/CNT@PU strain sensor exhibited high sensitivity and large strain range [37]. On one hand, the two-dimensional sheet-like GNPs could quickly slip to provide high sensitivity for the sensor when it was stretched [38]. On the other hand, the easily entangled one-dimensional CNTs connected the GNPs to ensure the stability of the conductive network structure under large tensile strains [39]. As is shown in Fig. 4c, the strain detection range and sensitivity of the CNT/GNP/CNT@PU are much higher compared with a series of piezoresistive sensors in recently reported literature [1-3,6,7,14,15,18,22]. Additionally, the CNT/GNP/CNT@PU strain sensor exhibited excellent sensitivity at a low strain ( $\mathrm{GF}=762$ at $5 \%$ strain), which is far superior to many reported studies [10,11]. From Fig. 4 d and Fig. S3, the sponge-based strain sensors can also respond to different frequencies $(0.01 \sim 1 \mathrm{~Hz})$. The $\Delta \mathrm{R} / \mathrm{R}_{0}$ of the sensors at the same strain of $10 \%$ increased with increasing tensile speed, showing a frequency dependence. A decrease in molecular mobility at high frequencies leads to mechanical response stiffness, which may be the main reason of frequency dependence [21]. All the sponge-based strain sensors can be used to identify different tensile strains (from 0.05 to $30 \%$ ), as shown in Fig. 4e. Due to its more complete conductive network, the CNT/GNP/CNT@PU sensor has better repeatability during cyclic stretching, even under a large strain (30\%). In addition, it is shown in Fig. 4f that the CNT/GNP/CNT@PU strain sensor has a lower response time $(\tau=37 \mathrm{~ms})$ at 10\% strain compared with the CNT@PU $(\tau=56 \mathrm{~ms})$ and CNT/GNP@PU $(\tau=42 \mathrm{~ms})$.

alt-text: Fig. 4

Fig. 4 

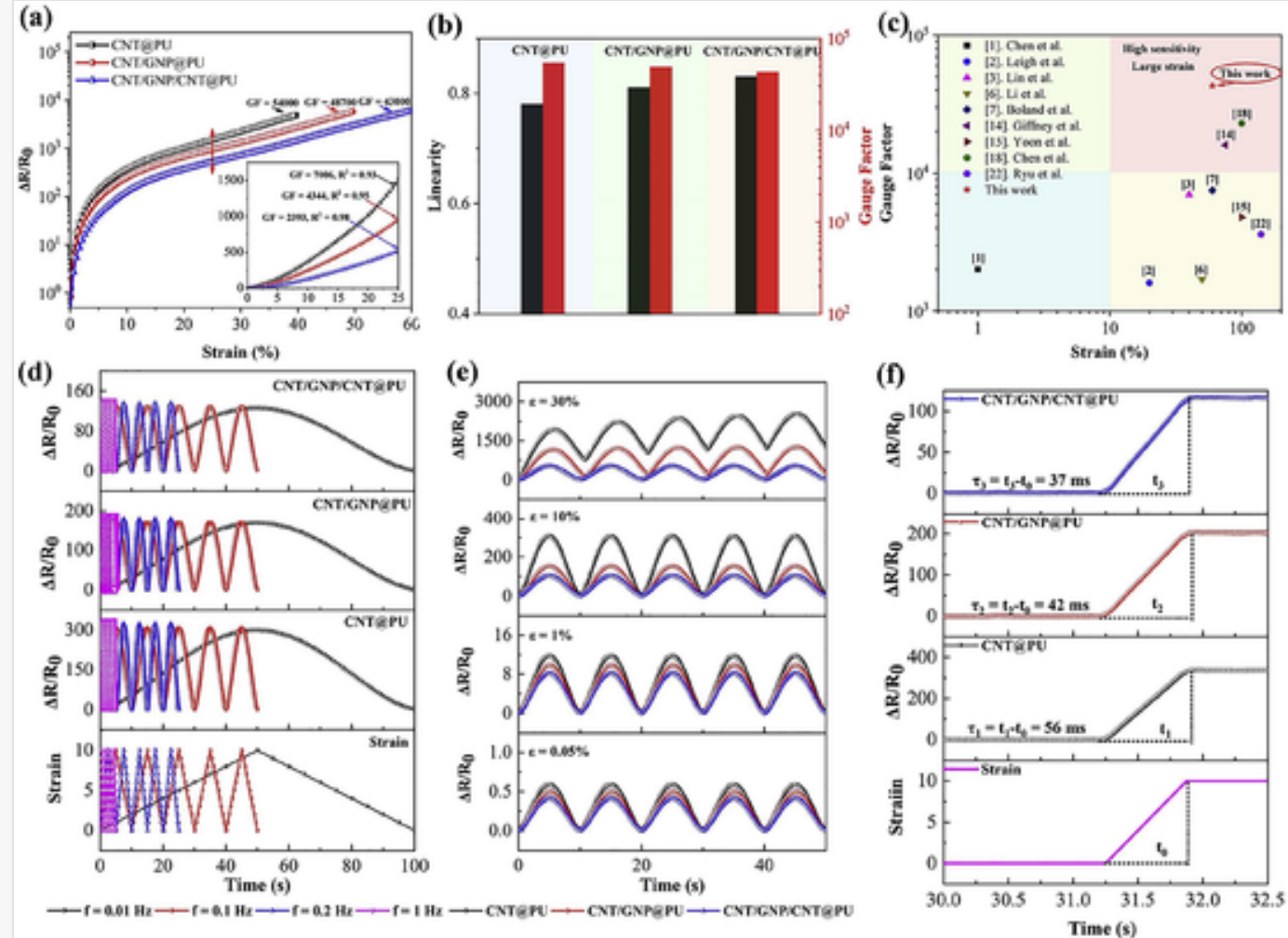

Sensing performance for tensile strain: (a) $\Delta \mathrm{R} / \mathrm{R}_{0}$-strain curves for the sponge-based sensors, (b) linearity and sensitivity of the sensors ( $\varepsilon>25 \%$ ), (c) GF value and workable strain-sensing range of the CNT/GNP/CNT@TPU strain sensor compared with those recently reported in literature, (d) $\Delta \mathrm{R} / \mathrm{R}_{0}$-frequency curves for the sponge-based sensors at the same strain $(\varepsilon=10 \%)$, (e) $\Delta \mathrm{R} / \mathrm{R}_{0}$ of the strain sensors under cyclic loading at different strains $(0.05 \%, 1 \%, 10 \%, 30 \%)$ and a frequency of $0.1 \mathrm{~Hz}$, (f) response time at $10 \%$ strain.

To verify the stability of the sponge-based sensors under tensile strain, they were cyclically stretched/released for 3000 times at a strain of $30 \%$ and a frequency of $0.1 \mathrm{~Hz}$ (Fig. 5a-c). The conductive nanoparticles in the sensors would be reoriented along the stretching direction, leading to simutaneous destruction and reconstruction of the conductive network [40,41]. The resistance of the CNT@PU and CNT/GNP@PU strain sensors increased significantly during the loading cycles. This indicates that the destruction of conductive network dominated the repeated stretching/releasing process, and the conductive network was irreversibly damaged. However, the CNT/GNP/CNT@PU showed much better stability during cyclic loading due to the more robust conductive network $[42,43]$. 
(a)

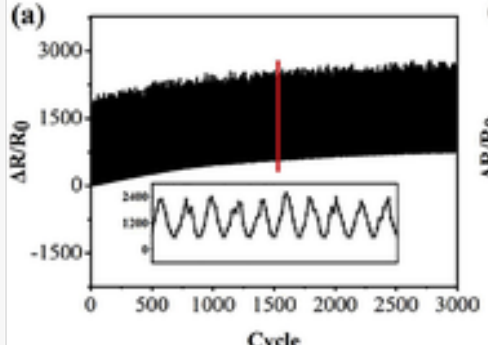

(d)

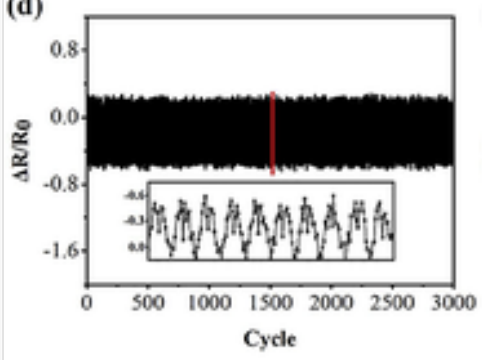

(b)

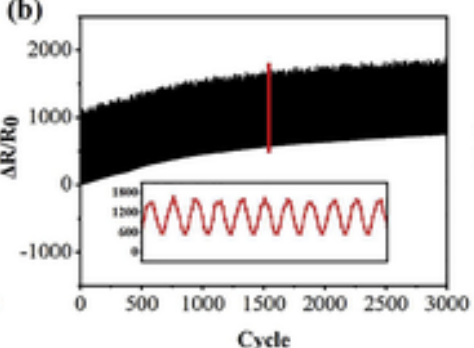

(e)

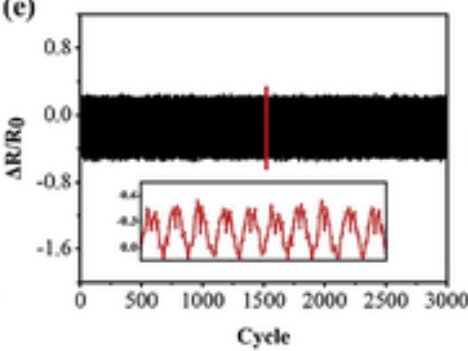

(c)

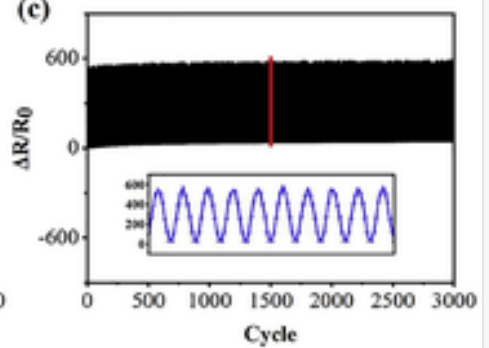

(f)

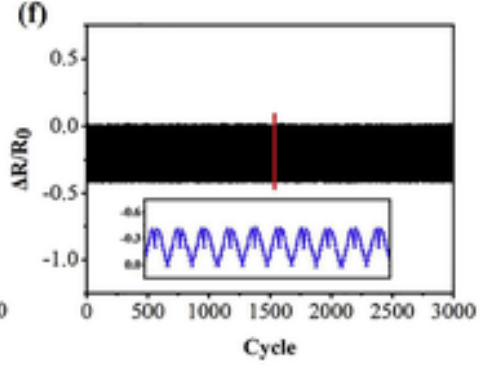

The stability of the strain sensors during 3000 stretching/releasing cycles at a strain of $30 \%$ and a frequency of $0.1 \mathrm{~Hz}$ : (a) CNT@PU, (b)CNT/GNP@PU, (c)CNT/GNP/CNT@PU; The stability of the strain sensors during 3000 compressing/releasing cycles at a strain of $30 \%$ and a frequency of $0.1 \mathrm{~Hz}$ : (d) CNT@PU, (e)CNT/GNP@PU, (f) CNT/GNP/CNT@PU.

\subsection{Sensing performance for compressive strain}

As is shown in Fig. 6a, the sponge-based strain sensors can be also used to monitor compressive strains from 0 to 50\%. The CNT@PU exhibited excellent sensitivity (GF =-2.1 at 20\% strain and GF =-1.3 at 50\% strain) and moderate linearity $\left(\mathrm{R}^{2}=0.95\right.$ at $0 \sim 20 \%$ strain and $\mathrm{R}^{2}=0.84$ at $20 \sim 50 \%$ strain), as shown in Fig. $6 \mathrm{~b}$. Reelectrostatic assembly improved the conductive network on the surface of sponge and enhanced its ability to resist deformation. Although the sensitivity ( $\mathrm{GF}=-1.8$ at $20 \%$ strain and $\mathrm{GF}=-1.1$ at $50 \%$ strain) of the CNT/GNP/CNT@PU sensor was slightly reduced compared with the CNT@PU, it showed higher linearity $\left(\mathrm{R}^{2}=0.98\right.$ at $0 \sim 20 \%$ strain and $\mathrm{R}^{2}=0.93$ at $20 \sim 50 \%$ strain). In general, all the sponge-based strain sensors have good linearity during compression, but the linearity of the CNT/GNP/CNT@PU is still higher than that of CNT@PU and CNT/GNP@PU. From Fig. 6c, the strain detection range and sensitivity of CNT/GNP/CNT@PU under compression are also much higher compared with a series of piezoresistive sensors recently reported in the literature $[5,8,16,24-27,1719][5,8,16,24-27,17,19]$. Additionally, the CNT/GNP/CNT@PU strain sensor exhibited ultra-high sensitivity at very low strain (GF = -12 at $0.05 \%$ strain), which is far superior to those reported in current literature [23-26]. From Fig. 6d and Fig. S4, the sponge-based strain sensors can also respond to different frequencies $(0.01 \sim 1 \mathrm{~Hz})$. The relative resistance changes of the sensors at the same strain of $10 \%$ increased with increasing compression speed, showing a frequency dependence. All the sponge-based strain sensors can be used to identify different compressive strains (from 0.05 to $30 \%$ ), as shown in Fig. 6e. The double peak in the $\Delta \mathrm{R} / \mathrm{R}_{0}$ curves during cyclic loading may be attributed to mechanical hysteresis during compression (Fig. S5) [18]. In addition, it can be observed in Fig. $6 \mathrm{f}$ that the CNT/GNP/CNT@PU strain sensor has a lower response time $(\tau=31 \mathrm{~ms})$ at $10 \%$ strain compared to the CNT/GNP@PU ( $\tau=36 \mathrm{~ms})$ and CNT@PU $(\tau=47 \mathrm{~ms})$. 

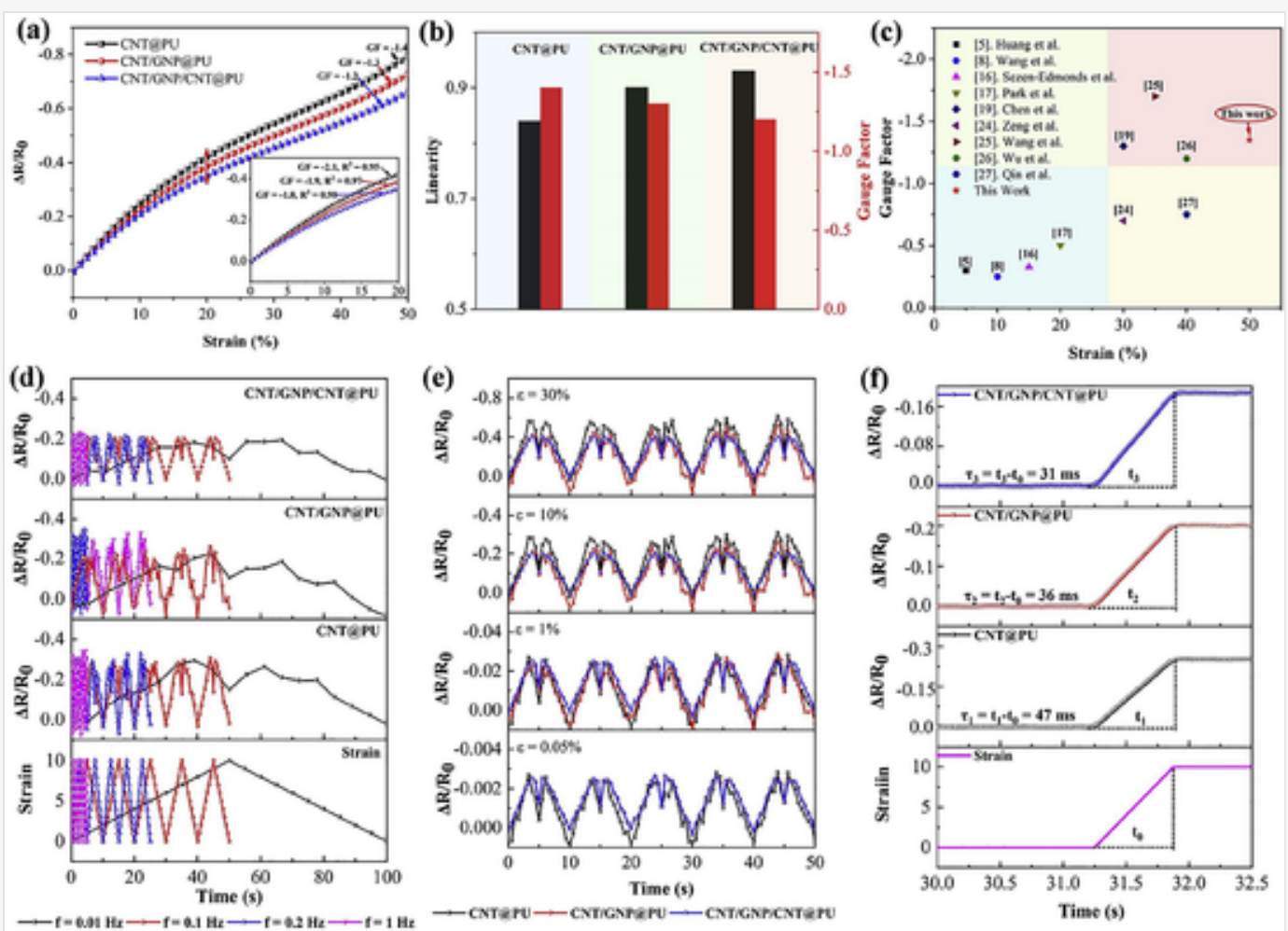

Sensing performance for compressive strain: (a) $\Delta \mathrm{R} / \mathrm{R}_{0}$-strain curves for the sponge-based sensors, (b) linearity and sensitivity of the sensors $(\varepsilon>20 \%$ ), (c) GF value and workable strain-sensing range of the CNT/GNP/CNT@TPU strain sensor compared with those recently reported in literature, (d) $\Delta \mathrm{R} / \mathrm{R}_{0}$-frequency curves for the sponge-based sensors at the same strain $(\varepsilon=10 \%)$, (e) $\Delta \mathrm{R} / \mathrm{R}_{0}$ of the strain sensors under cyclic loading at different strains $(0.05 \%, 1 \%, 10 \%, 30 \%)$ and a frequency of $0.1 \mathrm{~Hz}$, (f) response time at $10 \%$ strain.

To verify the stability of the sponge-based sensors under compressive strain, they were cyclically compressed/released for 3000 times at a strain of $30 \%$ and a frequency of $0.1 \mathrm{~Hz}$ (Fig. $5 \mathrm{~d}-\mathrm{f}$ ). All the spongebased strain sensors exhibited good stability and repeatability during the compression cycles. In particular, the $\Delta \mathrm{R} / \mathrm{R}_{0}$ of CNT/GNP/CNT@PU is more stable than that of CNT@PU and CNT/GNP@PU from Fig. 5d-f, indicating that the conductive network of the CNT/GNP/CNT@PU has higher resistance to destruction during the compression cycles.

\subsection{Applications}

Due to the high sensitivity and ability to recognize different strains such as stretching and compression, the CNT/GNP/CNT@PU sensor can be used to monitor different physiological activities of human body (Fig. 7). From Fig. 7a, when it was attached to the index finger using a medical tape, the $\Delta R / R_{0}$ of the strain sensor changed corresponding to the different bending angles of finger. Attaching the sensor to the wrist, the resistance of the sensor increased as the wrist was bent and quickly recovered after the wrist was straightened (Fig. 7b). It can also be used to identify the changes in the weight of water in a measuring cylinder when attached to a glove (Fig. 7c). The pressure on the finger increased with the increasing mass of water, and the resistance of the sponge-based strain sensor decreased. When the sensor was attached on the fingertip, it could also be used for Morse code compilation by detecting the touching and tapping signals. As shown in Fig. 7d, when the finger tapped the Morse codes of specific characters (i.e., "UKR.“), the real-time recorded signals could be used for information delivery after decryption by a code translator. When the sensor was fixed on the sole, it can be seen in Fig. 7e that it exhibited good signal responses to the feet movements. When the foot touched the ground, the resistance of the sensor reduced due to compression. After lifting the foot, the resistance of the sensor returned to the initial value $\left(\mathrm{R}_{0}\right)$ immediately. 

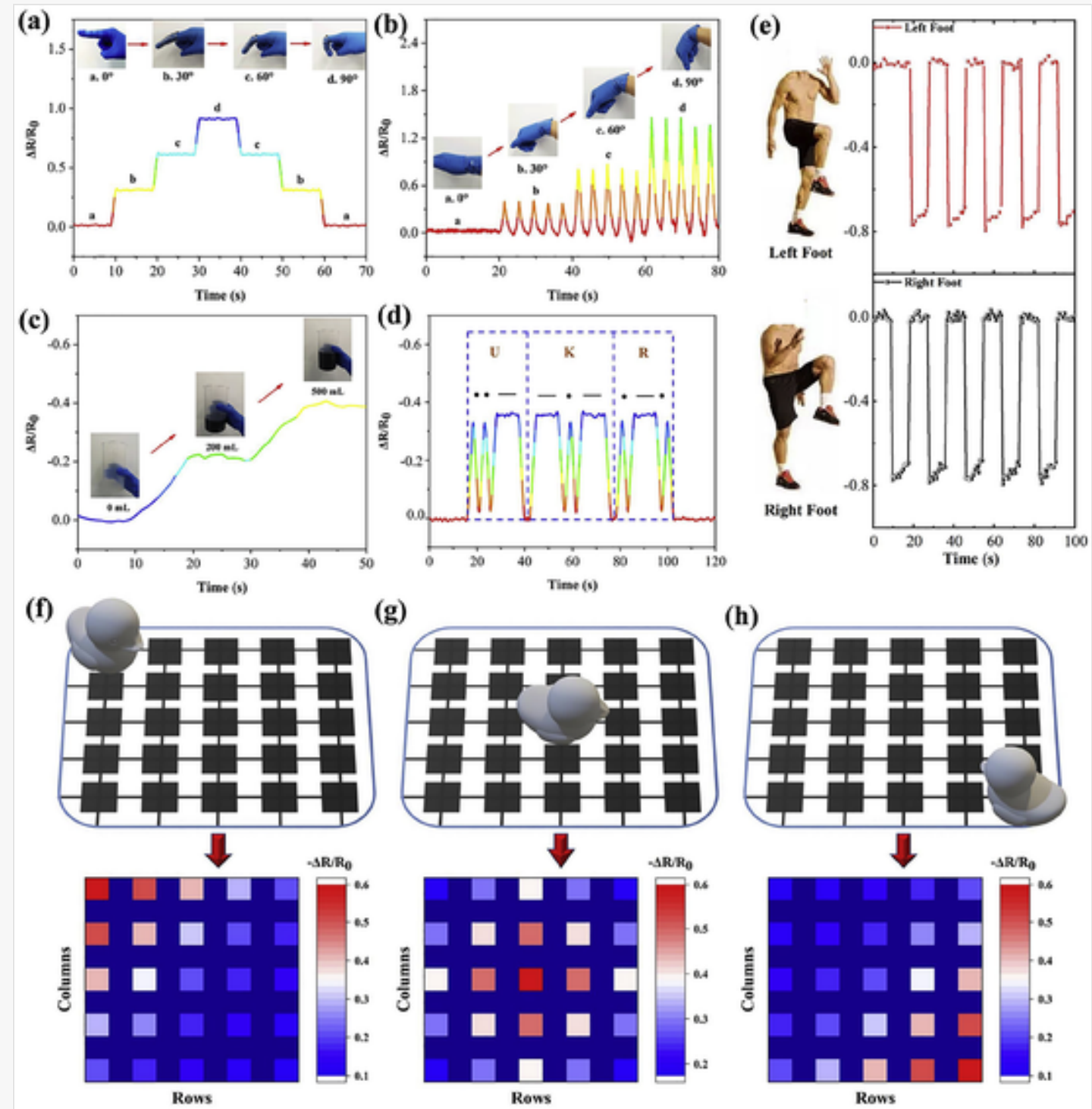

(g)

(h)
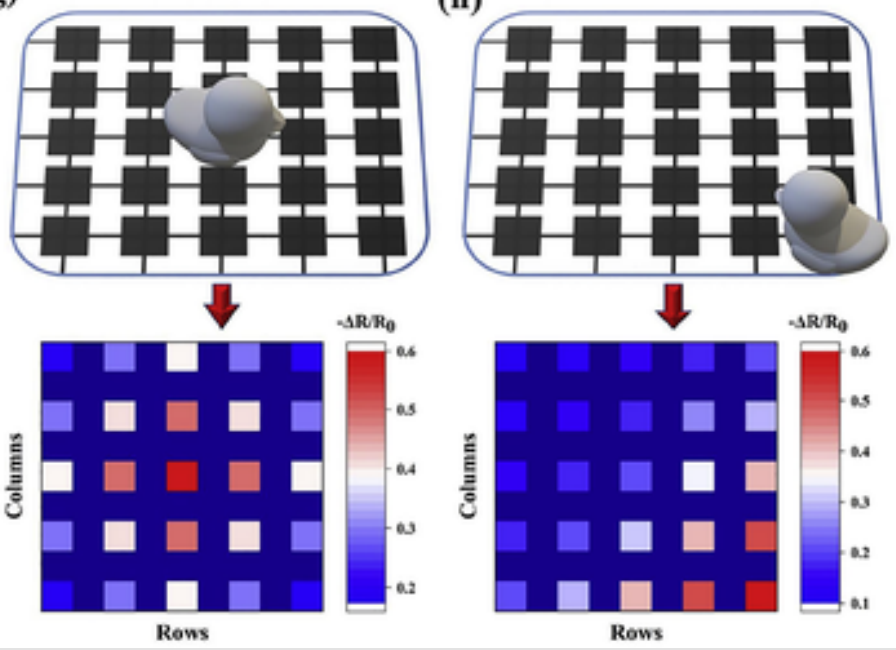

Electromechanical responses of the sensor to (a) finger bending, (b) wrist bending, (c) holding a container, (d) signal output for Morse codes “UKR" and (e) walking; (f-h) a sensor array based on the CNT/GNP/CNT@PU sensors for accurate location identification of an object.

The highly sensitive sponge-based strain sensor can be also used to prepare a sensor array to monitor the location of an object [32]. A $5 \times 5$ sensor array made of the CNT/GNP/CNT@PU sensors $(10 \mathrm{~mm} \times 10 \mathrm{~mm})$ with a distance of $10 \mathrm{~mm}$ was embedded in a foam substrate. A small yellow duck of $5.62 \mathrm{~g}$ was placed in different locations of the array (Fig. 7f-h). Due to the changes in location of the duck, the resistance of the sponge-based strain sensor also changed. The resistance of some sensors decreased due to compression (the largest decrease in resistance exactly occurred at the location of yellow duck), while the resistance of sensors in other areas was almost constant, demonstrating the ability of the sensor array to identify pressure magnitude and position.

\section{Conclusions}

In this work, we prepared a flexible and high-performance strain sensor by simple layer-by-layer electrostatic self-assembly of carbon nanoparticles on commercial polyurethane (PU) sponges. The synergistic effect between the CNTs and GNPs contributes to the construction of a more complete conductive network, which improves the electrical conductivity of the modified sponges. The more complete conductive network helps to improve the 
ability of sponge-based strain sensors to resist destruction, which gives the CNT/GNP/CNT@PU sensor a higher linearity and repeatability compared with the CNT@PU and CNT@PU sensors. The CNT/GNP/CNT@PU strain sensor exhibited excellent piezoresistive behavior under both compressive and tensile strains with high sensitivity $(\mathrm{GF}=43,000$ at $60 \%$ tensile strain and $\mathrm{GF}=-1.1$ at $50 \%$ compressive strain), high linearity $\left(\mathrm{R}^{2}=0.83\right.$ at tensile strain over $25 \%, \mathrm{R}^{2}=0.93$ at compressive strain over $\left.20 \%\right)$ and outstanding stability under cyclic loading (>3000 cycles). Additionally, the CNT/GNP/CNT@PU strain sensor has the ability to monitor different frequencies $(0.01 \sim 1 \mathrm{~Hz})$ and low response time $(\tau=37 \mathrm{~ms}$ at $10 \%$ tensile strain and $\tau=31 \mathrm{~ms}$ at $10 \%$ compressive strain). Thanks to its excellent performance, the sponge-based strain sensor can be used to monitor the physiological activities of human body, including finger movements, wrist bending and walking. In addition, a $5 \times 5$ sensor array using the sponge-based strain sensors was prepared for accurate location identification of an object. This work provides a simple strategy to prepare high-performance, multi-purpose sponge-based strain sensors with potential applications in intelligent robots, prosthetics and wearable devices.

\section{Author Statement}

Dong Xiang: made the equal contributions to this work, including the conception and design of this work, analysis and interpretation of the data and writing of the manuscript. All the persons who have made substantial contributions to this work have been authored in the manuscript. Xuezhong Zhang: made the equal contributions to this work, including the conception and design of this work, analysis and interpretation of the data and writing of the manuscript. All the persons who have made substantial contributions to this work have been authored in the manuscript. Yongfeng Zheng: participated in the preparation and partial tests of the sensors. All the persons who have made substantial contributions to this work have been authored in the manuscript. Wanqiu Zhu: participated in the preparation and partial tests of the sensors. All the persons who have made substantial contributions to this work have been authored in the manuscript. Eileen Harkin-Jones: provided substantial contributions in interpreting the data and editing the manuscript, All the persons who have made substantial contributions to this work have been authored in the manuscript. Ping Wang: provided substantial contributions in interpreting the data and editing the manuscript. Chunxia Zhao: provided substantial contributions in interpreting the data and editing the manuscript. All the persons who have made substantial contributions to this work have been authored in the manuscript. Hui Li: provided substantial contributions in interpreting the data and editing the manuscript. All the persons who have made substantial contributions to this work have been authored in the manuscript. Bin Wang: provided substantial contributions in interpreting the data and editing the manuscript. Yuntao Li: provided substantial contributions in interpreting the data and editing the manuscript. All the persons who have made substantial contributions to this work have been authored in the manuscript.

\section{Declaration of competing interest}

The authors declare that they have no known competing financial interests or personal relationships that could have appeared to influence the work reported in this paper.

\section{Acknowledgements}

This work is supported by International Cooperation Project of Chengdu (2019-GH02-00054-HZ), Scientific Research Starting Project of SWPU (2019QHZ011), Open Experimental Program of SWPU (KSZ19514), Undergraduate Innovation Program of Sichuan Province (201910615027), and Scientific Research Foundation for the Returned Overseas Chinese Scholars of Sichuan Province. 
Supplementary data to this article can be found online at https://doi.org/10.1016/j.compscitech.2020.108437.

\section{References}

The corrections made in this section will be reviewed and approved by a journal production editor. The newly added/removed references and its citations will be reordered and rearranged by the production team.

[1] Chen S., Wei Y., Wei S., et al., Ultrasensitive cracking-assisted strain sensors based on silver nanowires/graphene hybrid particles, ACS Appl. Mater. Interfaces 8 (2016) 25563-25570.

[2] Leigh S.J., Bradley R.J., Purssell C.P., et al., A simple, low-cost conductive composite material for 3D printing of electronic sensors, PLoS One 7 (2012) 1-6.

[3] Lin L., Liu S., Zhang Q., et al., Towards tunable sensitivity of electrical property to strain for conductive polymer composites based on thermoplastic elastomer, ACS Appl. Mater. Interfaces 5 (2013) 5815-5824.

[4] Begley M.R., Gianola D.S., Ray T.R., Bridging functional nanocomposites to robust macroscale devices, Science 364 (2019) 4299-4311.

[5] Huang W., Dai K., Zhai Y., et al., Flexible and lightweight pressure sensor based on carbon nanotube/thermoplastic polyurethane-aligned conductive foam with superior compressibility and stability, ACS Appl. Mater. Interfaces 9 (2017) 42266-42277.

[6] Li Y., Samad Y.A., Taha T., et al., Highly flexible strain sensor from tissue paper for wearable electronics, ACS Sustain. Chem. Eng. 4 (2016) 4288-4295.

[7] Boland C.S., Khan U., Backes C., et al., Sensitive, high-strain, high-rate bodily motion sensors based on graphene-rubber composites, ACS Nano 8 (2014) 8819-8830.

[8] Wang X., Meng S., Tebyetekerwa M., et al., Highly sensitive and stretchable piezoresistive strain sensor based on conductive poly(styrene-butadiene-styrene)/few layer graphene composite fiber, Compos Part A Appl Sci Manuf 105 (2018) 291-299.

[9] Zhou K., Zhao Y., Sun X., et al., Ultra-stretchable triboelectric nanogenerator as high-sensitive and self-powered electronic skins for energy harvesting and tactile sensing, Nanomater. Energy 70 (2020) 104546-104555.

[10] Ren F., Song D., Li Z., et al., Synergistic effect of graphene nanosheets and carbonyl iron-nickel alloy hybrid filler on electromagnetic interference shielding and thermal conductivity of cyanate ester composites, J. Mater. Chem. C 6 (2018) 1476-1486.

[11] Yue X., Jia Y., Wang X., et al., Highly stretchable and durable fiber-shaped strain sensor with porous core-sheath structure for human motion monitoring, Compos. Sci. Technol. 189 (2020) $108038-108045$. 
[12] Zheng Y., Qin J., Shen J., et al., Controllable distribution of conductive particles in polymer blends via bilayer structure design: a strategy to fabricate shape-memory composites with tunable electroresponsive properties, J. Mater. Chem. C 8 (2020) 9593-9601.

[13] Hu C., Li Z., Wang Y., Gao J., et al., Comparative assessment of the strain-sensing behaviors of polylactic acid nanocomposites: reduced graphene oxide or carbon nanotubes, J. Mater. Chem. C 5 (2017) 2318-2328.

[14] Giffney T., Bejanin E., Kurian A.S., et al., Highly stretchable printed strain sensors using multiwalled carbon nanotube/silicone rubber composites, Sensors Actuators, A Phys 259 (2017) 44-49.

[15] Yoon Y., Samanta K., Lee H., et al., Highly stretchable and conductive silver nanoparticle embedded graphene flake electrode prepared by in situ dual reduction reaction, Sci. Rep. 5 (2015) $1-10$.

[16] Sezen-Edmonds M., Khlyabich P.P., Loo Y.L., Tuning the magnitude and the polarity of the piezoresistive response of polyaniline through structural control, ACS Appl. Mater. Interfaces 9 (2017) 12766-12772.

[17] Park H., Kim J.W., Hong S.Y., et al., Microporous polypyrrole-coated graphene foam for highperformance multifunctional sensors and flexible supercapacitors, Adv. Funct. Mater. 28 (2018) 111.

[18] Chen Q., Xiang D., Wang L., et al., Facile fabrication and performance of robust polymer/carbon nanotube coated spandex fibers for strain sensing, Compos Part A Appl Sci Manuf 112 (2018) 186-196.

[19] Chen Q., Li Y., Xiang D., Zheng Y., et al., Enhanced strain sensing performance of polymer/carbon nanotube-coated spandex fibers via noncovalent interactions, Macromol. Mater. Eng. 305 (2020) 1-10.

[20] Gong T., Zhang H., Huang W., et al., Highly responsive flexible strain sensor using polystyrene nanoparticle doped reduced graphene oxide for human health monitoring, Carbon 140 (2018) 286-295.

[21] Wang L., Chen Y., Lin L., et al., Highly stretchable, anti-corrosive and wearable strain sensors based on the PDMS/CNTs decorated elastomer nanofiber composite, Chem. Eng. J. 362 (2019) 89-98.

[22] Ryu S., Lee P., Chou J.B., et al., Extremely elastic wearable carbon nanotube fiber strain sensor for monitoring of human motion, ACS Nano 9 (2015) 5929-5936.

[23] Hao B., Mu L., Ma Q., Yang S., Ma P., Stretchable and compressible strain sensor based on carbon nanotube foam/polymer nanocomposites with three-dimensional networks, Compos. Sci. Technol. 163 (2018) 162-170.

[24] Zeng Z., Seyed Shahabadi S.I., et al., Highly stretchable, sensitive strain sensors with a wide linear sensing region based on compressed anisotropic graphene foam/polymer nanocomposites, Nanoscale 9 (2017) 17396-17404. 
[25] Wang L., Xiang D., Harkin-Jones E., et al., A flexible and multipurpose piezoresistive strain sensor based on carbonized phenol formaldehyde foam for human motion monitoring, Macromol. Mater. Eng. 304 (2019) 1-9.

[26] Wu X., Han Y., Zhang X., et al., Large-area compliant, low-cost, and versatile pressure-sensing platform based on microcrack-designed carbon Black@Polyurethane sponge for human-machine interfacing, Adv. Funct. Mater. 26 (2016) 6246-6256.

[27] Qin Y., Peng Q., Ding Y., et al., Lightweight, superelastic, and mechanically flexible graphene/polyimide nanocomposite foam for strain sensor application, ACS Nano 9 (2015) 89338941.

[28] Xiang D., Wang L., Tang Y., et al., Effect of phase transitions on the electrical properties of polymer/carbon nanotube and polymer/graphene nanoplatelet composites with different conductive network structures, Polym. Int. 67 (2018) 227-235.

[29] Kim J.Y., Ji S., Jung S., et al., 3D printable composite dough for stretchable, ultrasensitive and body-patchable strain sensors, Nanoscale 9 (2017) 11035-11046.

[30] Zhang Q., Zhang F., Xu X., et al., Three-dimensional printing hollow polymer template-mediated graphene lattices with tailorable architectures and multifunctional properties, ACS Nano 12 (2018) 1096-1106.

[31] Cataldi P., Ceseracciu L., Marras S., et al., Electrical conductivity enhancement in thermoplastic polyurethane-graphene nanoplatelet composites by stretch-release cycles, Appl. Phys. Lett. 110 (2017) 121904-121908.

[32] Lu Y., Biswas M.C., Guo Z., et al., Recent developments in bio-monitoring via advanced polymer nanocomposite-based wearable strain sensors, Biosens. Bioelectron. 123 (2019) 167-177.

[33] Xiao Z., Sheng C., Xia Y., et al., Electrical heating behavior of flexible thermoplastic polyurethane/Super-P nanoparticle composite films for advanced wearable heaters, J. Ind. Eng. Chem. 71 (2019) 293-300.

[34] Liu H., Li Y., Dai K., et al., Electrically conductive thermoplastic elastomer nanocomposites at ultralow graphene loading levels for strain sensor applications, J. Mater. Chem. C 4 (2015) 157166.

[35] Christ J.F., Aliheidari N., Ameli A., et al., 3D printed highly elastic strain sensors of multiwalled carbon nanotube/thermoplastic polyurethane nanocomposites, Mater. Des. 131 (2017) 394-401.

[36] Muth J.T., Vogt D.M., Truby R.L., et al., Embedded 3D printing of strain sensors within highly stretchable elastomers, Adv. Mater. 26 (2014) 6307-6312.

[37] Liu H., Gao J., Huang W., et al., Electrically conductive strain sensing polyurethane nanocomposites with synergistic carbon nanotubes and graphene bifillers, Nanoscale 8 (2016) $12977-12989$.

[38] Mondal S., Khastgir D., Elastomer reinforcement by graphene nanoplatelets and synergistic improvements of electrical and mechanical properties of composites by hybrid nano fillers of 
graphene-carbon black \& graphene-MWCNT, Compos. Part A Appl Sci Manuf. 102 (2017) 154165.

[39] Peng X., Wu K., Hu Y., et al., A mechanically strong and sensitive CNT/rGO-CNF carbon aerogel for piezoresistive sensors, J. Mater. Chem. 6 (2018) 23550-23559.

[40] Xiang D., Zhang X., Li Y., et al., Enhanced performance of 3D printed highly elastic strain sensors of carbon nanotube/thermoplastic polyurethane nanocomposites via non-covalent interactions, Compos. B Eng. 176 (2019) 107250-107261.

[41] Xiang D., Zhang X., Harkin-Jones E., et al., Synergistic effects of hybrid conductive nanofillers on the performance of $3 \mathrm{D}$ printed highly elastic strain sensors, Compos Part A Appl Sci Manuf 129 (2020) 105730-105741.

[42] Lu Y., Chandra M., Guo Z., et al., Wujcik, Biosensors and Bioelectronics Recent developments in bio-monitoring via advanced polymer nanocomposite-based wearable strain sensors, Biosens. Bioelectron. 123 (2019) 167-177.

[43] Ye X., Yuan Z., Tai H., et al., A wearable and highly sensitive strain sensor based on a polyethylenimine-rGO layered nanocomposite thin film, J. Mater. Chem. C 5 (2017) 7746-7752.

\section{Graphical abstract}

alt-text: Image 1

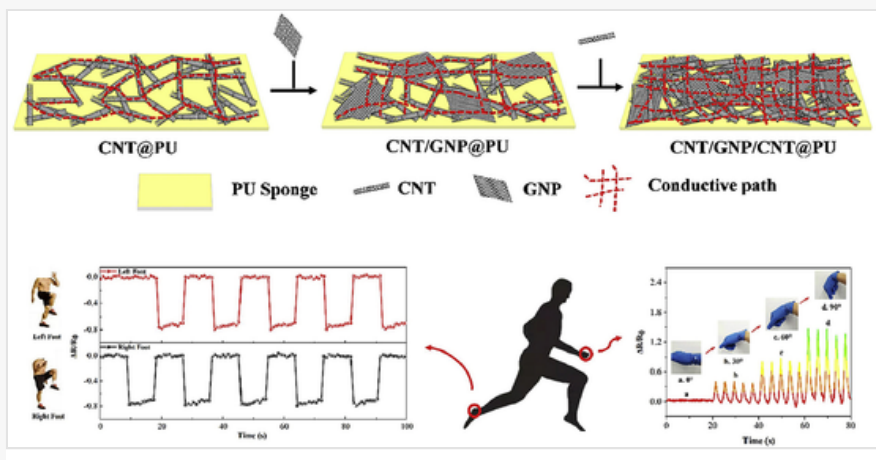

\section{Appendix ASupplementary data}

The following is the Supplementary data to this article:

\section{$\underline{\text { Multimedia Component } 1}$}

\section{Multimedia component 1}

alt-text: Multimedia component 1 
Query: Your article is registered as a regular item and is being processed for inclusion in a regular issue of the journal. If this is NOT correct and your article belongs to a Special Issue/Collection please contact n.thandavamoorthy@elsevier.com immediately prior to returning your corrections.

Answer: Yes

Query: Please confirm that given names and surnames have been identified correctly and are presented in the desired order and please carefully verify the spelling of all authors' names.

Answer: Yes

Query: Please confirm that the provided emails “dxiang01@hotmail.com, yuntaoli@swpu.edu.cn” are the correct address for official communication, else provide an alternate e-mail address to replace the existing one, because private e-mail addresses should not be used in articles as the address for communication.

Answer: We confirm that the email adresses are correct.

Query: Have we correctly interpreted the following funding source(s) and country names you cited in your article: Research Foundation, United States; International Cooperation, European Union; SWPU, China?

Answer: The content of acknowledgements and funding information are correct. All the fundings are from China. 